\title{
FRACTURE-DISLOCATION OF THE SHOULDER WITH INTERPOSITION OF LONG HEAD OF BICEPS
}

\author{
Report of a Case
}

\author{
R. S. Henderson, London, ENGland
}

From the Orthopaedic Department, St Bartholomew's Hospital, London

A foundry worker, aged thirty, underwent electro-convulsive therapy for a depressive disorder. Curare was not used. After the treatment his shoulder became swollen, painful and stiff. Radiographs showed a subcoracoid fracture-dislocation with separation of an exceptionally large fragment of the greater tuberosity (Fig. 1). Two days later, under general anaesthesia, the head of the humerus was guided back into the glenoid fossa without much difficulty. Radiographs in the theatre, however, showed that a large gap still separated it from the arulsed fragment, and manipulations, including full abduction, failed to improve the apposition (Fig. 2 ). Open reduction was therefore decided upon.

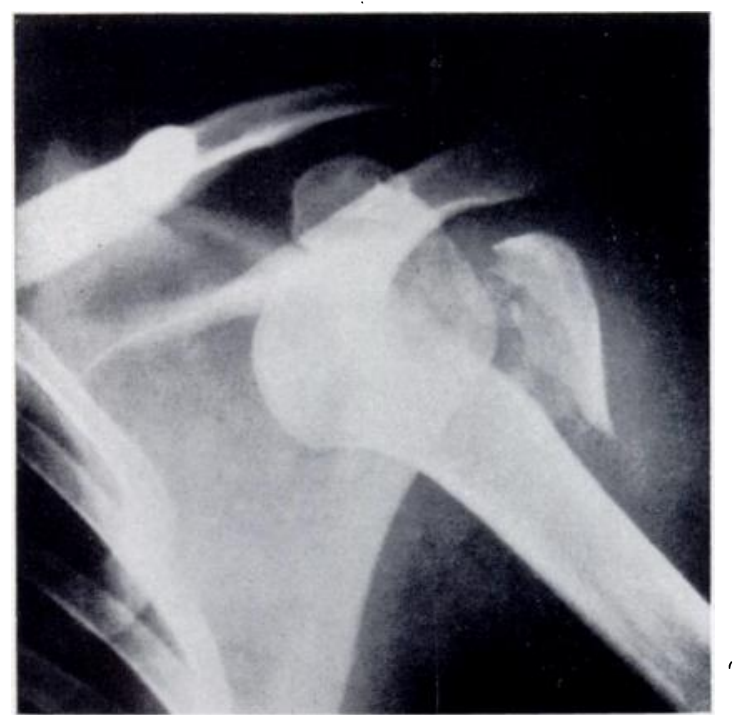

FIG. 1

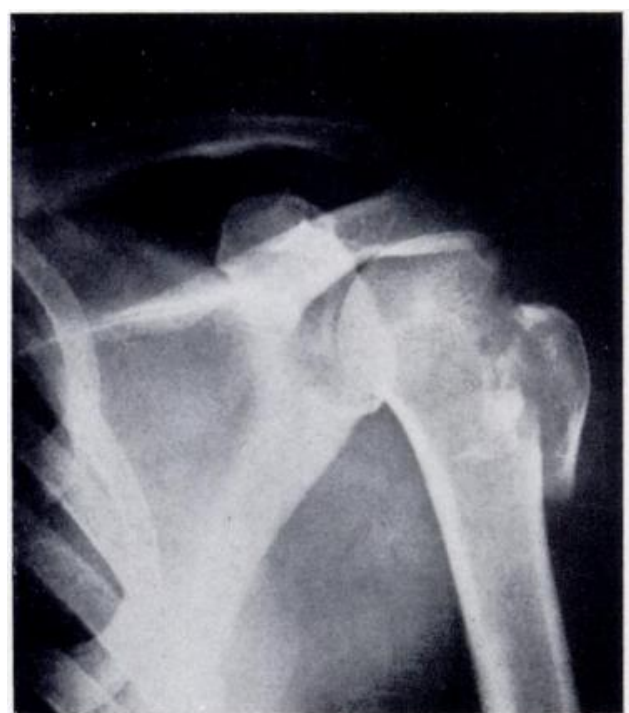

FIG. 2

Figure 1-Radiograph showing fracture-dislocation of shoulder before manipulation. Figure 2-Radiograph showing persistently displaced fragment of greater tuberosity after manipulation.

At operation, through a deltoid-splitting incision, the anterior part of the capsule was found to be completely disrupted. The articular surfaces were in their normal relationship with each other but the fragment was discovered lying behind the joint in a position of full lateral rotation. Between it and what remained of the intact head lay the tendon of the long head of biceps, effectively preventing reduction (Fig. 3). When the tendon was held aside, it was possible to pull the fragment forwards with a bone hook and rotate it medially into its correct position. The attached supraspinatus cuff at the same time returned to its normal relationship with the joint. As the fragment seemed unstable, it was fixed with two screws.

Active movements were started ten days after operation; two months later, rotation and abduction were half normal and the patient was able to return to work. 
Comment-Though many injuries have occurred during electro-convulsive therapy without the administration of curare, fracture-dislocation of the shoulder is perhaps one of the less common. Cornell (1943) described a case of fracture-dislocation of both shoulders in an eclamptic seizure; both were reduced by manipulation. Meyerding (1937), reviewing a series

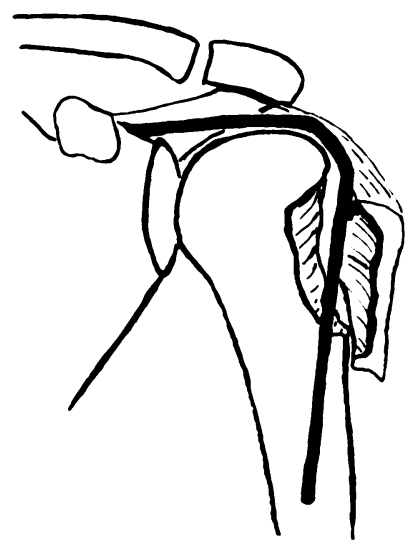

Fig. 3

Diagram showing interposition of tendon of long head of biceps between head of humerus and fragment of greater tuberosity.

The fracture line corresponds approximately to the bicipital groove.

of dislocated shoulders, found that it per cent of the subcoracoid type were associated with fractures of the greater tuberosity. He described one such injury with rupture of the biceps tendon. De Palma (1950) mentions that the biceps tendon may mechanically impede reduction.

It must be noted that electro-convulsive therapy may produce unusual types of injury because of the exceptional violence of contractions of opposing muscle groups.

I should like to thank Mr H. Jackson Burrows for his help and permission to publish this case, and Mr R. (i. Whitley of the Institute of Orthopaedics for his help with the illustrations.

\section{REFERENCES}

(Oonveli., E. I.. (1943): Fracture and Dislocation of Both Shoulders as a Result of Eclamptic Seizure. American Journal of Obstetrics and Gynecology, 45, 354.

DE PALMA, A. F. (1950): Surgery of the Shoulder. Philadelphia: J. B. Lippincott Company.

Meyerding, H. W. (1937): Fracture-I)islocation of the Shoulder. Minnesota Medicine, 20, 717. 